\title{
Silent Sinus Syndrome: Clinical Findings and Differential Diagnosis
}

\author{
Paolo Bossolesi ${ }^{*}$, Barbara Pedruzzi2*, Enzo Emanuelli ${ }^{3}$ \\ ${ }^{1}$ U.O. Otorinolaringoiatria Azienda ULSS 8 Asolo, Ospedale di Castelfranco Veneto e Montebelluna (TV), Italy \\ ${ }^{2}$ U.O.C. Otorinolaringoiatria e Otochirurgia, Azienda Ospedaliera di Padova, Padova, Italy \\ ${ }^{3}$ U.O.A. Chirurgia Endoscopica delle Vie Aeree, Azienda Ospedaliera di Padova, Padova, Italy \\ Email: ${ }^{*}$ paolo.bossolesi@ulssasolo.ven.it ${ }^{*}$ barbarengy@hotmail.com
}

Received 6 March 2014; revised 5 April 2014; accepted 2 May 2014

Copyright (C) 2014 by authors and Scientific Research Publishing Inc.

This work is licensed under the Creative Commons Attribution International License (CC BY).

http://creativecommons.org/licenses/by/4.0/

c) (i) Open Access

\section{Abstract}

The Silent Sinus Syndrome (SSS) is a rare condition that causes facial asymmetry, unilateral enophthalmos and diplopia. It is thought to be secondary to chronic maxillary sinus atelectasis (CMA) with reabsorbed bone and subsequent displacement of the orbital floor. Such anatomic modifications occur over time, and therefore it is possible to encounter different stages of the same disease with or without orbital displacement. Clinical findings can be unclear so it makes sense to recognize potentially evolving SSS while other disturbances have to be ruled out. Our purpose is to underline clinical findings for different diagnosis and proper management. We consider Functional Endoscopic Sinus Surgery (FESS) indicated in CMA and SSS to halt the progression of the disease. Nevertheless restitution treatment of enophtalmos due to orbital floor displacement involves plastic reconstruction of the floor of the orbit via transconjunctival approach. We report a case of SSS and discuss distinctive features of non-neoplastic lesions involving the maxillary sinus that should be considered for differential diagnosis.

\section{Keywords}

Silent Sinus Syndrome, SSS, Unilateral Enophthalmos, Diplopia, Chronic Maxillary Sinus Atelectasis, Displacement of the Orbital Floor, Functional Endoscopic Sinus Surgery

\section{Introduction}

The maxillary sinus is present at birth, undergoing further expansion with age. Two main points of rapid growth

${ }^{*}$ Corresponding authors. 
occur, from birth until the age of 3 years and then from 7 years age through early adolescence. The sinus then slowly grows until it reaches adult size by the age of 18 years. Later in adult life, the sinus continues to pneumatize inferiorly and may expand to contain tooth roots. The average size of the maxillary sinus is $34 \mathrm{~mm} \times 33 \mathrm{~mm}$ $\times 23 \mathrm{~mm}$, with an average volume of $15 \mathrm{~mL}$. In adults, the floor of the maxillary sinus can be below the level of the nasal floor. The maxillary sinus is housed in the body of the maxilla, with the inferior orbital wall as the superior border, the lateral nasal wall as the medial boundary, the alveolar process of the maxilla as its inferior border, and the canine fossa as the anterior border. The maxillary sinus outflow tract is the ostiomeatal complex, which is collectively constituted by the uncinate process, the maxillary ostium, the infundibulum, and the ethmoid bulla. These structures form a functional complex through which the maxillary sinus contents egress. Relief of the ostiomeatal complex in case of its obstruction is the principle of FESS. The uncinate process is a sickle-shaped very thin bone that starts anterosuperiorly and slopes posteroinferiorly. It has its anterior attachment along the frontal process of the maxilla and lacrimal bone and free edge in its superior and dorsal surface so that is placed along the lateral nasal wall in a flap like position defining a solid angle opened backwards. Superiorly the uncinate process may attach to the lamina papyracea (most common configuration), the middle turbinate, or the skull base. At its most posterior point it attaches to the inferior turbinate at the ethmoidal process. The ethmoid bulla lies just posterior to the uncinate, it is the most constant anterior ethmoid air cell. The infundibulum is the opening between the ethmoidal bulla and the free edge of the uncinate process including the space that lies underneath delimited lateral to the medial wall of the maxillary sinus. The natural maxillary sinus ostium is placed lateral to the uncinate process within the ethmoid infundibulum. The cilia of the epithelium that lines the maxillary sinus beat uphill toward the natural maxillary sinus ostium.

SSS is characterized by painless involution of maxillary sinus after occlusion of the ethmoid infundibulum; associated enophthalmos differentiate the syndrome versus CMA alone. This condition appears to be always unilateral and affects adult patients in their third to fifth decade of life. The presenting symptom is spontaneous one side enophthalmos rising slightly over the course of several months, even if rare cases of sudden oneset are also described [1]. Mild nose disturbances may be present, but the patient's main complaint always regards some aesthetic and uncomfortable changing in her appearance like eyelid retraction, superior orbital sulcus deepening or unpleasant flattening of the malar region.

Eye movements and visual function may be affected and gaze diplopia may occur [2]. CT scan and MR imaging of the orbits and sinuses demonstrate unilateral maxillary sinus opacification and collapse, with inferior bowing of the orbital floor. Office nasal endoscopic examination carried on by mean of a 30 degree angled $2.7 \mathrm{~mm} \phi$ endoscope in basal condition and after decongestion of nasal mucosa is the best mean to clearly identify pathological changing in the nasal lateral wall anatomy, furthermore it is mandatory to the surgeon for operation planning [3].

The constant findings in all the patients with SSS are:

- Normal or mild inflammated nasal mucosal lining.

- Enlarged middle meatus.

- Uncinate process completely adherent to the lateral wall obstructing maxillary natural ostium.

- No pathologic secretion along the ethmoido-maxillary drainage pathway.

CT has proven to be precious giving details on bone shape and thickness with clear anatomic picture constantly showing the following features:

- Diminished volume of maxillary antrum with retraction of all or most walls.

- Compensatory augmentation in ipsilateral orbital volume.

- Complete opacification of affected sinus.

- Lateralized uncinated process.

- Demineralization of sinus walls.

- Expanded retroantral fat pad.

MR enforces the evidence of CT imaging showing:

- Opacified sinus with mixed signal contents and diminished volume (T1WI).

- Prominence of orbital fat and widening of the retroantral fat pad (T1 \& T2WI).

- Mixed signal central secretions with high signal peripheral thick oedematous mucosal lining (T2WI).

Since such anatomic modifications occur over time, therefore it is possible to encounter different stages of the same disease with or without orbital displacement. Clinical findings can be unclear so it makes sense to recognize potentially evolving SSS while other disturbances have to be ruled out. In this article, our purpose is to underline clinical findings for different diagnosis and proper management. We report a case of SSS and discuss distinctive 
features of non-neoplastic lesions involving the maxillary sinus that should be considered for differential diagnosis.

\section{Case Reports}

Case 1: Silent Sinus Syndrome

An otherwise healthy 34-year-old man became aware of progressive left side deepening of the superior orbital sulcus causing visible asymmetry of the face; he also referred a one-year history of chronically constipated nose. The physician recommended an MR scan and than sent him to an ENT specialist who suspected chronic sinusitis and scheduled the patient for a Caldwell-Luc procedure then the patient claimed for further evaluation to our institution. His appearance was affected with notable left enophtalmos (Figure 1). He complained about the aesthetic defect, he denied any nose and sinus major disturbances or facial pain. Under evaluation of the ophthalmologist a mild deficit of elevation in left eye with contralateral hyperfunction appeared. Diplopia in the left upper sight position was evoked, downward dislocation of the left eye bulb with about $2 \mathrm{~mm}$ enophthalmos reported. CT showed no relevant septal deviation, complete opacification of the left side maxillary sinus with inferior bowing of the floor of the orbit. Remarkable reduction in the medio-lateral and caudo-cranial diameter of the antrum compared to the contra lateral was present as consequence of demineralised posterior and superior walls collapse. Retroantral fat pad was enlarged. The middle turbinate was upright with no other sinus involvement. The uncinate process was in close contact with the lateral wall being also bent inward and smashed
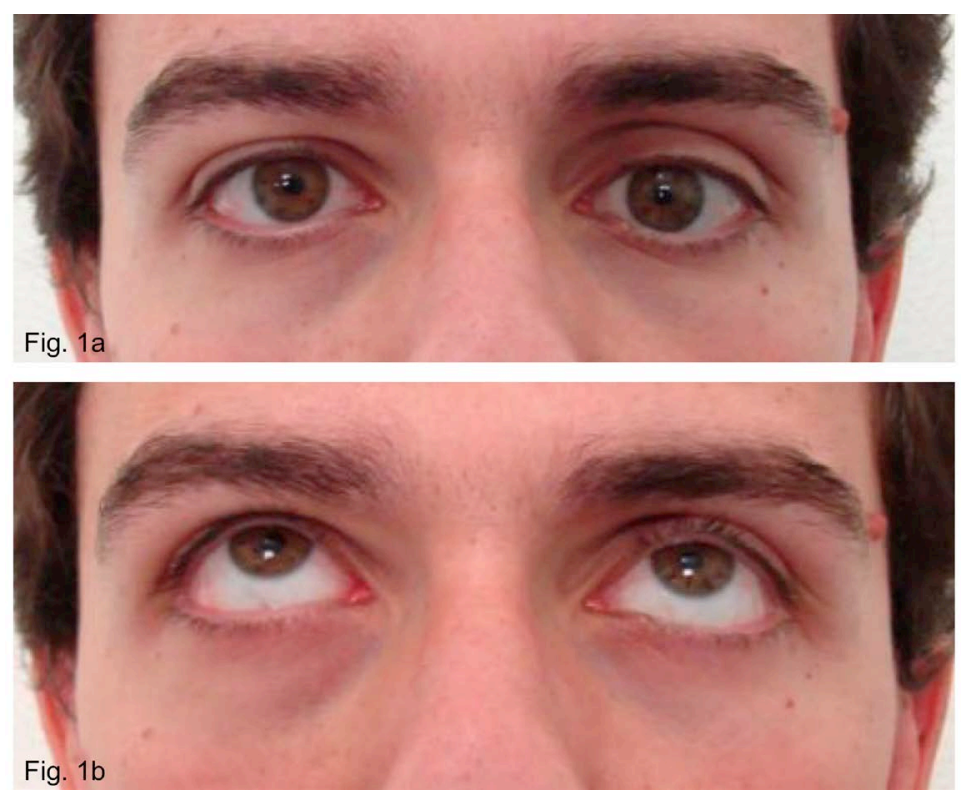

Figure 1. (a) Left side $2 \mathrm{~mm}$ enophthalmos with superior orbital sulcus deepening. (b) The same in the upward sight.

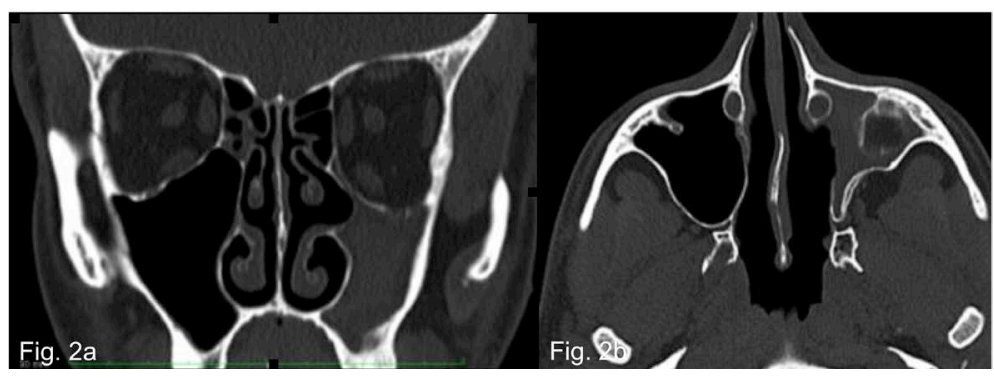

Figure 2. (a) (b) Diminished volume of left maxillary antrum with retraction of medial, posterior and superior walls. Compensatory augmentation in ipsilateral orbital volume. Complete opacification of affected sinus. Lateralized uncinate process. 
against the orbital floor (Figure 2). MR findings showed prolapsed inferior aspect of the orbit, retraction of posterior wall of the antrum, reduced volume filled with secretions content (Figure 3). Office nasal endoscopyshowed no septal deformity, normal mucosal nasal lining, enlarged middle meatus with uncinate process completely adherent to the lateral wall impairing the patency of maxillary sinus natural ostium. He was treated with endoscopic antrostomy to free the ethmoido-maxillary drainage pathway, thick mucous secretion was removed form the sinus cavity and sent for microbiological examination: no bacterial growth was seen. The patient refused reconstruction of the floor of the orbit via transconjunctival approach for aesthetic purpose. After one year the patient is asymptomatic, follow-up postoperative MR shows healthy unblocked left maxillary sinus, no further remodelling of bone occurred over time nor the sinus regained his original shape (Figure 4).

The inclusion criteria to establish the diagnosis of SSS include [3]:

- Spontaneous enophthalmos secondary to inferior bowing of osteopenic thin orbital floor with fully developed opacified maxillary sinus.

- Absence of major sinus pathologies as nasal polyps, cysts, fungus ball or acute rhinosinusitis in the last 6 months.

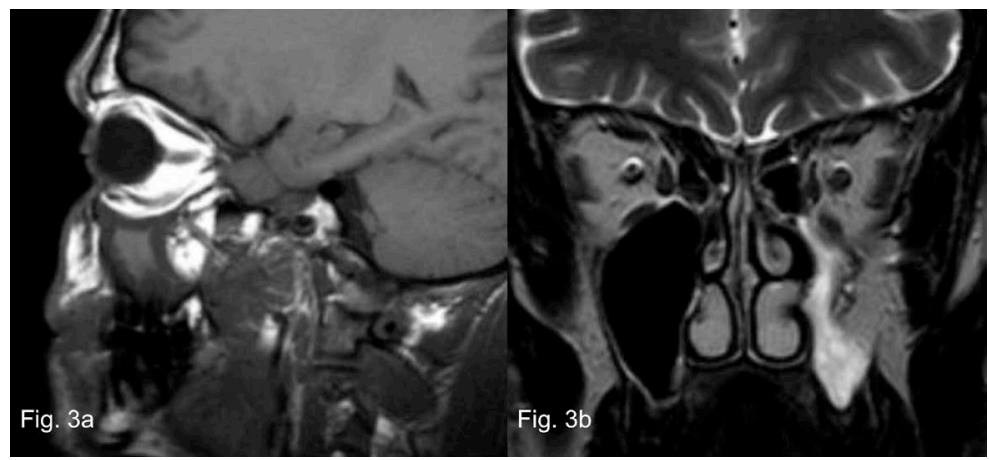

Figure 3. (a) MR imaging shows opacified maxillary sinus with mixed signal contents and diminished volume (T1WI). (b) Hyperintense secretion content into the left maxillary sinus (T2WI).

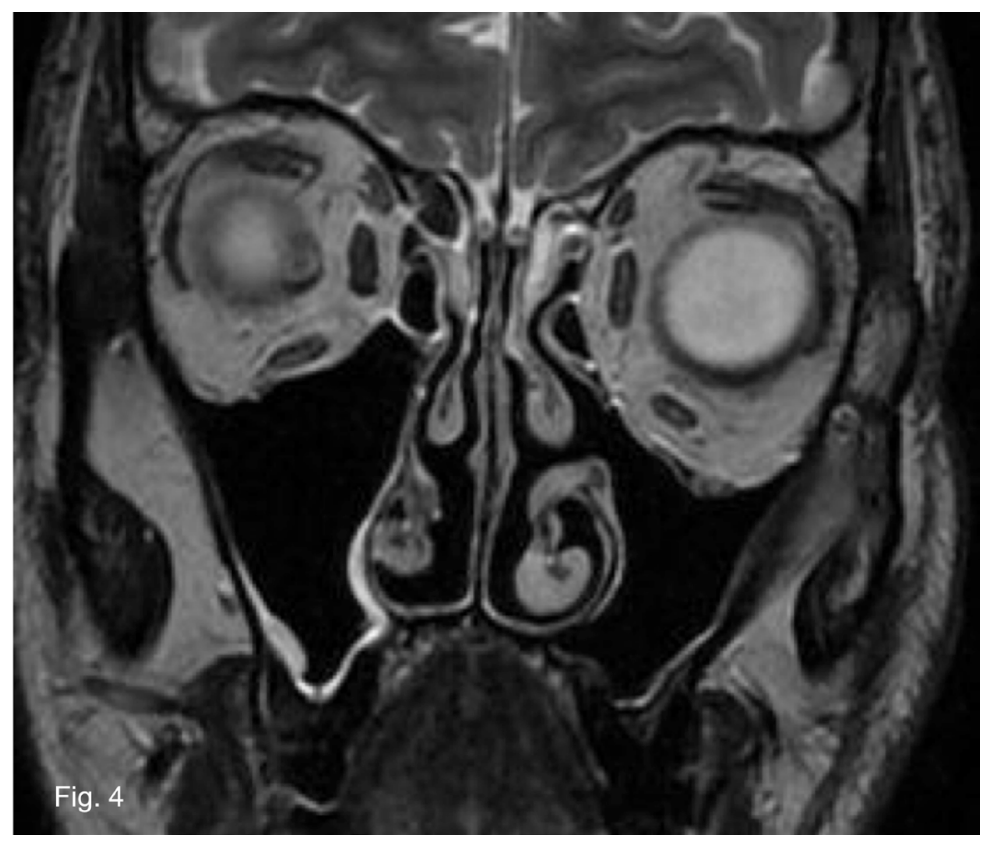

Figure 4. Two year postoperative MRI control, the left maxillary sinus is airfilled with normal mucosal lining and patent drainage pathway, no further rearrangement of the bony boundaries took place. 
- Absence of previous trauma, surgery or other causes of acquired enophthalmos.

- Absence of congenital facial deformity [2] [4]-[9].

FESS is the way to re-establish the physiological drainage pathway and ventilation of maxillary sinus, usual safe technique to separate the uncinate process from backward is recommended. We performed a regular size antrostomy by mean of medial displacement and cutting of the lower uncinate process starting from its free posterior edge without scratching the mucosal lines. Freeing maxillary natural ostium enables to aspirate mucous secretions stuffed within the atelectasic sinus. Irrigation with saline sterile solution completed sinus wash-out. Specimens sent to lab for microbiological examination gave negative results confirming non-infective aetiology. We paid particular attention not to damage the lamina papyracea while raising the posterior boundary of the uncinate process adherent to the lateral nasal wall. This eventually bothersome manoeuvre that carries a risk of injury to the orbital content has been held on in a safe way with a blunt palpating hook.

The closest condition to SSS is CMA, usually these patients refer a history of chronic or recurrent nasal symptoms like stuffiness, discharge or less frequently facial-ache; nevertheless CMA can also be an incidental finding. All features are analogous to that of SSS but ocular involvement. CMA causes inward bowing of one or more antral walls, more likely the medial one. Thick mucous content fills the whole residual cavity, asymmetry of orbital floors may be found.

Case 2: CMA

A 57-year-old woman who suffered of episodic nasal congestion and headache always cured with symptomatic drugs underwent CT scan cause of a face trauma. After that also MR was scheduled and she was sent to ENT unit with suspect of chronic sinusitis (Figure 5). At examination with endoscopy widened middle meatus was seen, no secretion or other abnormality. She denied any visual discomfort or aesthetic changing in her appearance, she agreed with clinicians to proceed with FESS to free maxillary sinus drainage pathway.

The most frequent disturbance of the maxillary sinus is chronic sinusitis, often it is sustained by the presence of schizomicetes particularly aspergillus that forms a cluster filling the whole cavity, in the non immune-compromised patient this infections remains confined within the sinus and is not likely to extend in a tissue-aggressive fashion. Simple surgical wash out and air refilling of the antrum provides recovery.

Case 3: maxillary fungal ball sinusitis

A 57-year-old woman complains of right facial pain, unilateral runny nose with frequent mucopurulent discharge and cacosmia. She was previously diagnosed and treated with drugs for trigeminal neuralgia, for the same reason she underwent MR C+ scan. After that she went to attention. RM showed spin-ECO T2 weighed sequences with right maxillary sinus peripheral iperintensity and void signal into the lumen (Figure 6(c)), analogous pattern with $\mathrm{T} 1$ weighed sequences and $\mathrm{T} 1 \mathrm{C}+$ with peripheral gadolinium enhancement (Figure 6(d)). Preoperative CT scan confirmed the suspect of fungal ball sinusitis showing typical iron body like figure with opaque sinus content (Figure 6(a) and Figure 6(b)). Endoscopic antrostomy and drainage was successfully performed.

Isolated mucocele of the maxillary sinus is a relatively uncommon condition, it has a round shaped concentric growth based on enlargement under pressure of the mucosal lined encasement of mucoid secretion, the walls of the lesion often are the sinus walls themselves, surrounding bone is reabsorbed, the main difference between
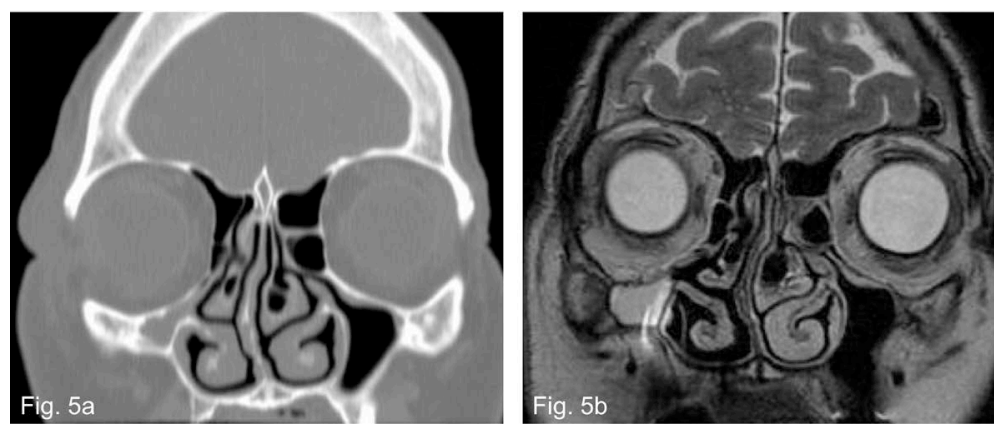

Figure 5. (a) (b) Right maxillary sinus chronic atelectasis, the anterior and medial walls of the antrum are displaced inward, the uncinate process is adherent to the lamina papyracea, the floor of the orbit is still in physiological shape and the eye is not affected. 


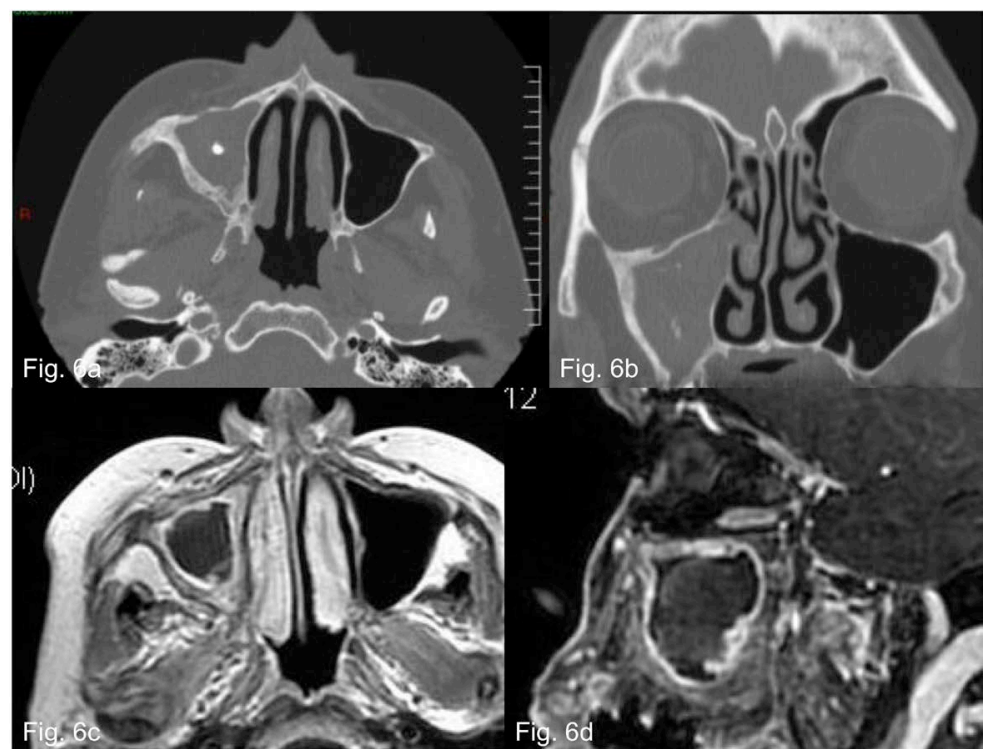

Figure 6. (a) CT scan in axial and (b) coronal view in right side chronic fungal maxillary sinusitis (fungus ball), the sinus is filled with fungal content. CT scan shows the distinctive radiolucent foreign body like aggregates (iron body like). (c) MRI shows mixed hypointense signal into the sinus (T1WI) in axial view. (d) The same MRI in sagittal view.

mucocele of the maxillary sinus and CMA is that mucocele is displacing outward the normal boundary of the sinus involved while CMA cause the sinus to shrink.

Case 4: maxillary mucocele

A 40-year-old man complains about pain in his right cheek and stuffy nose, sinusitis was diagnosed so he went on antibiotic course without any improvement. Further careful examination of CT scan showed a bone lined round shaped mass within the sinus lumen occupying the upper half and impairing the natural ostium (Figure 7). This finding was considered suggestive for mucocele arose from a Haller cell. FESS under loco-regional anaesthesia permitted marsupialization of the mucocele and restitution of normal sinus outflow.

The maxillary sinus is in close anatomic and embryologic relationship with the alveolar process of the maxilla so growing lesions of odontogenic origin are not uncommon. The most frequently encountered according also with data found in literature is periapical cyst also known as radicular cyst. A periapical (radicular) cyst is the most common odontogenic cyst. The usual aetiology is a tooth that becomes infected, leading to necrosis of the pulp. Periapical inflammation stimulates the Malassez epithelial rests resulting in the formation of a periapical granuloma. It may be infected or sterile and eventually becomes a cyst. Radiographically present as radiolucent lesions in association with the apex of a not vital tooth, RM T1 weighted C+ shows peripheral enhancement and proximity with the dental apex (Figure 8). Occasionally, these lesions can become quite large because they grow in response to pressure. Several treatment options exist for such cysts including FESS in selected cases. Many cysts resolve with endodontic therapy of the involved tooth, lesions that fail to resolve with such therapy should be surgically removed and histopathologically examined.

The second most common odontogenic cyst is the dentigerous cyst, which develops within the normal dental follicle that surrounds an unerupted tooth. These cysts can grow very large and cause surrounding bone remodelling. The usual radiographic appearance is that of a well demarcated lesion attached at an acute angle to the cervical area of an unerupted tooth (Figure 9).

Far less frequent is the primordial cyst; it develops presumably by the dental follicle that forms and subsequently undergoes cystic degeneration without ever completing odontogenesis. The histology of these lesions is a nondescript stratified squamous epithelium.

Case 5: odontogenic developmental cyst (primordial cyst)

Young male of 25 years old came to attention for persistent stuff nose and unpleasant sensation of nasal blockage in his right side. The physician saw a slightly deviated nasal septum therefore ordered a CT scan with the suspicion of chronic sinusitis. CT showed right maxillary sinus opacyfication with a star shaped intensely ra- 


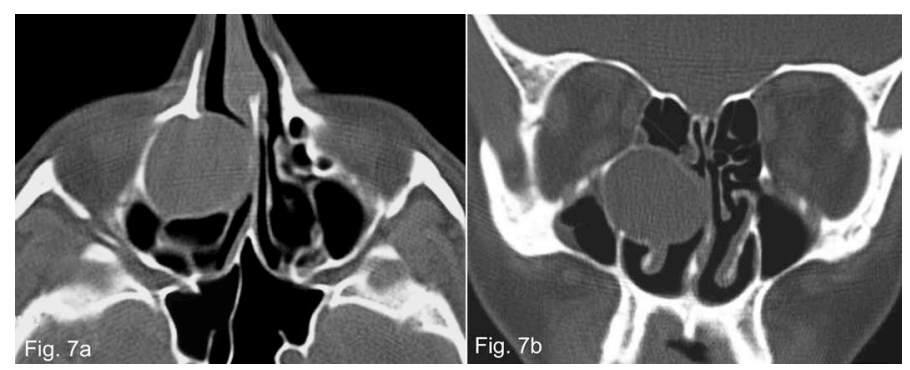

Figure 7. (a) (b) TC scan shows right maxillary sinus mucocele arose from the Haller cell.

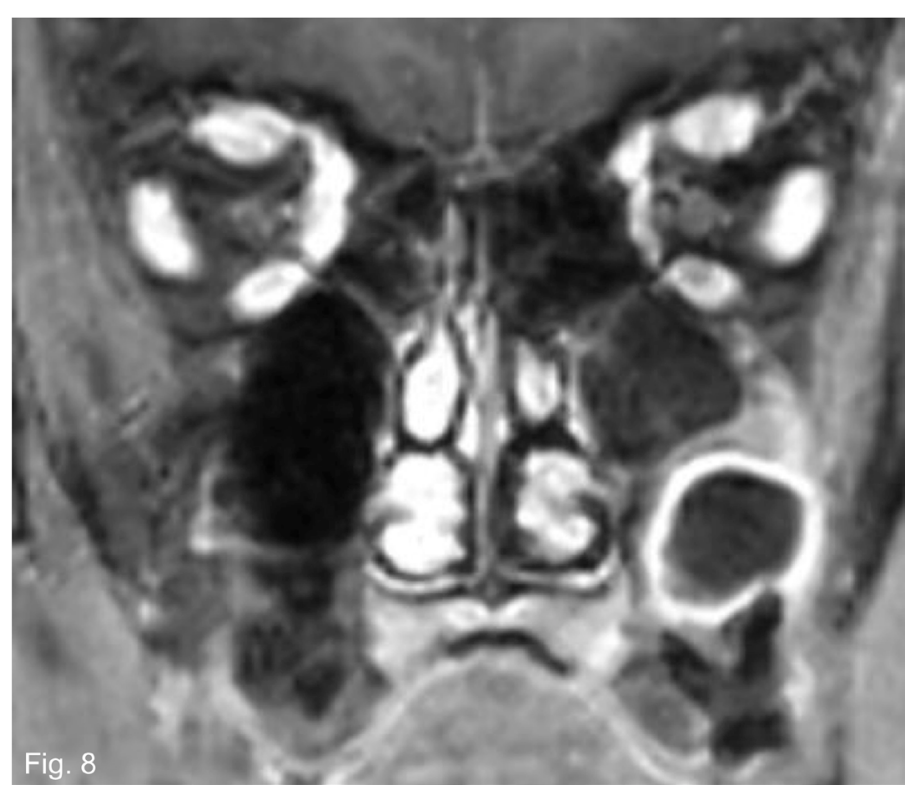

Figure 8. MRI showing peripheral enhancement of a periapical cyst affecting the left maxillary sinus.

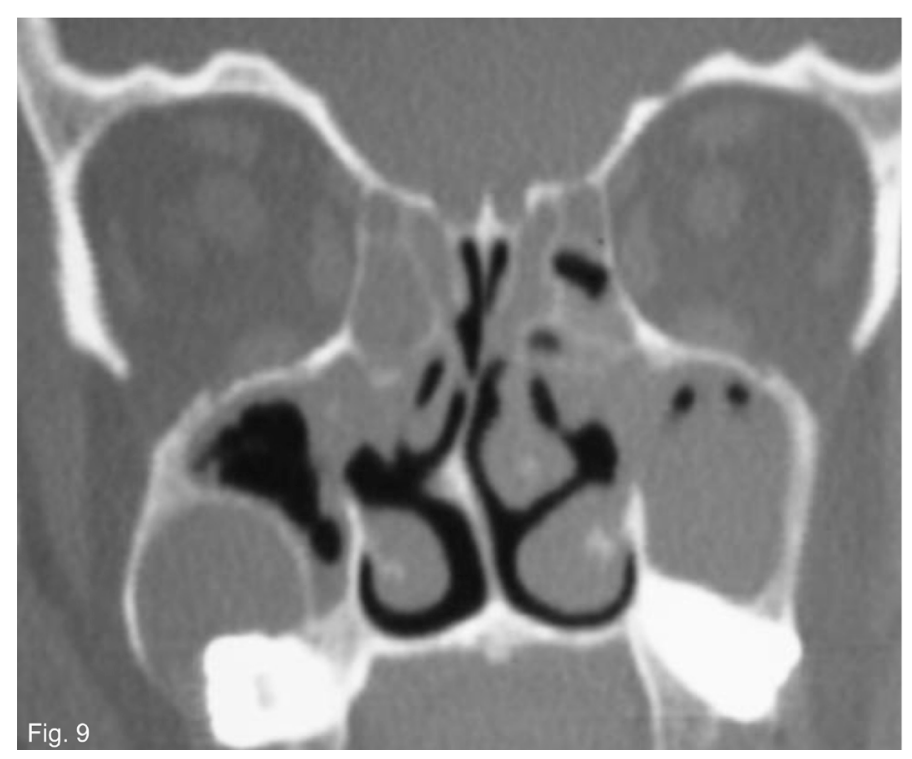

Figure 9. CT scan showing a dentigerous cyst of the third molar affecting left maxillary sinus. 
diopaque element within its upper posterior portion (Figure 10(c)). The patient was scheduled for surgery with the suspect of fungal sinusitis. MRI showed no contrast enhancement and T2 weighted brilliant iperintensity, absence of void signal in the sinus content (Figure 10(b)). Those findings didn't match with the diagnosis of fungus ball. Nevertheless the sinus was approached in local anaesthesia under endoscopic control. Medialization of the lateral nasal wall was apparent and once incised yellowish clear fluid draw off, a thin cyst wall was lining the internal maxillary sinus, and it was separated and sent for histopathologic examination. The patient recovered fast with no need for nasal stuffing, the maxillary sinus appears aerated with a normal mucosa at office endoscopic examination after 5 years follow-up.

Other conditions have to be considered in presence of a unilateral opacified poorly developed maxillary sinus: malformation especially in young individuals [10] [11] (Figure 11), trauma sequels and of course neoplasm.

\section{Discussion}

Silent sinus syndrome is a rare and therefore not always well-recognized disease that affects the maxillary sinus and finally the eye. Nevertheless several disturbances may result in maxillary sinus altered content and bony changing appreciable with imaging technology, because of that the differential diagnosis has to rule out developmental anomalies, neoplasm and endocrinological or immunological problems able to affect the maxillary sinus and orbit at one side. CT scan is nowadays included in routine sinus examination therefore accurate check of this region helps to differentiate causes of sinus opacification and bony boundaries alterations. Attention as to be

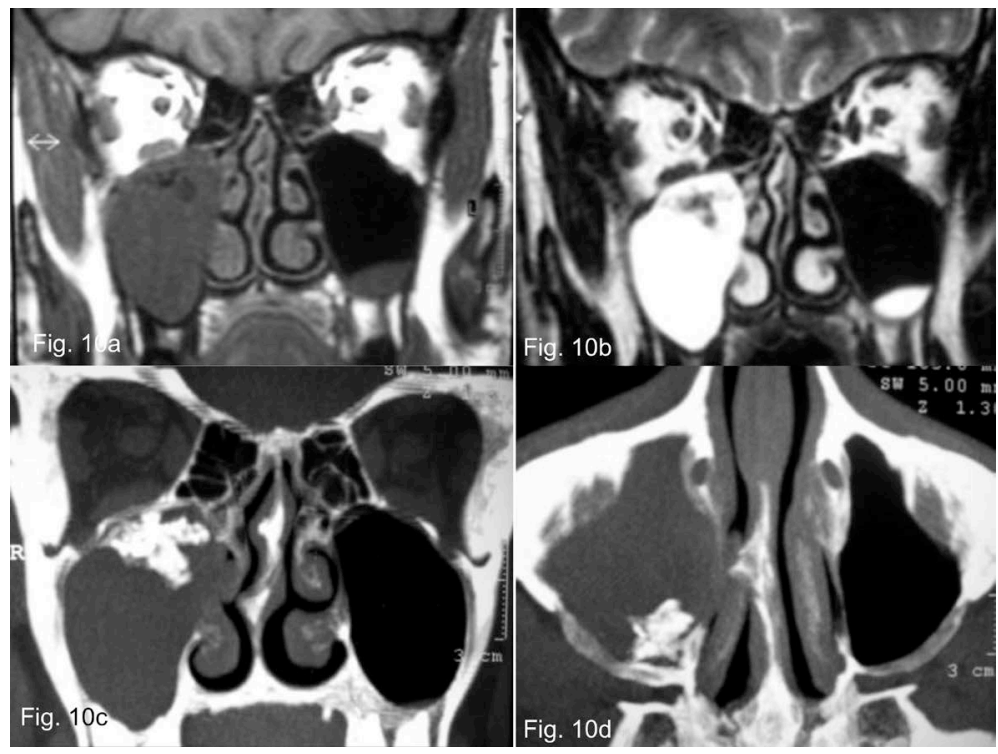

Figure 10. (a) and (b) Developmental odontogenic cyst (primordial cyst) of the right maxillary sinus. (c) and (d) Mineralized epithelium is evident as a star shaped CT radiolucent foreign body like image. (b) MRI shows hyperintense liquid content of the cyst that fills completely the maxillary sinus (T2WI).

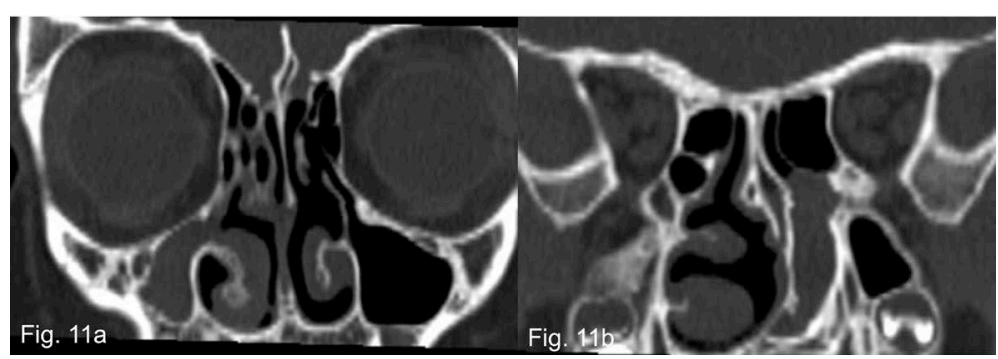

Figure 11. (b) Child with unilateral choanal atresia and (a) contra lateral atelectasic maxillary sinus. 
paid to the sinus and the orbit fine anatomy with regard on sinus development, volume, degree of aeration, wall configuration and appearance of the sinus infundibulum. Additional MRI is useful if CT appears to be doubtful or incomplete.

With CMA orbital floor falling downward is an inconstant feature but it bears critical relevance. The assumption that individuals affected might develop orbital displacement is accepted [12]-[15] but the exact sequence of events has not been established [8]. We encountered patients with CMA who never experienced any ocular or aesthetic disorder while imaging showed inward bowing of one or more antral walls, including initial deformation of orbital floor (Figure 12). Sometimes the shrinkage of the sinus involves just the medial wall (Figure 13). Nevertheless a different degree of pneumatisation and symmetry in maxillary sinus is often observable also in the healthy population [12] and extreme conditions may resemble CMA or SSS for example an underdeveloped cavity within an almost completely bone structured maxilla (Figure 14) or a tiny sinus with normal air

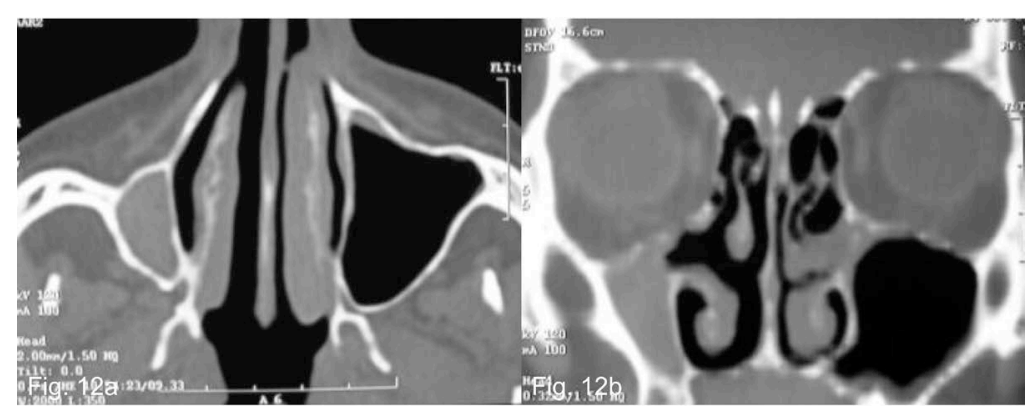

Figure 12. (a) CT scan of a case of CMA showing inward bowing of anterior and medial antral walls, (b) including initial deformation of orbital floor.

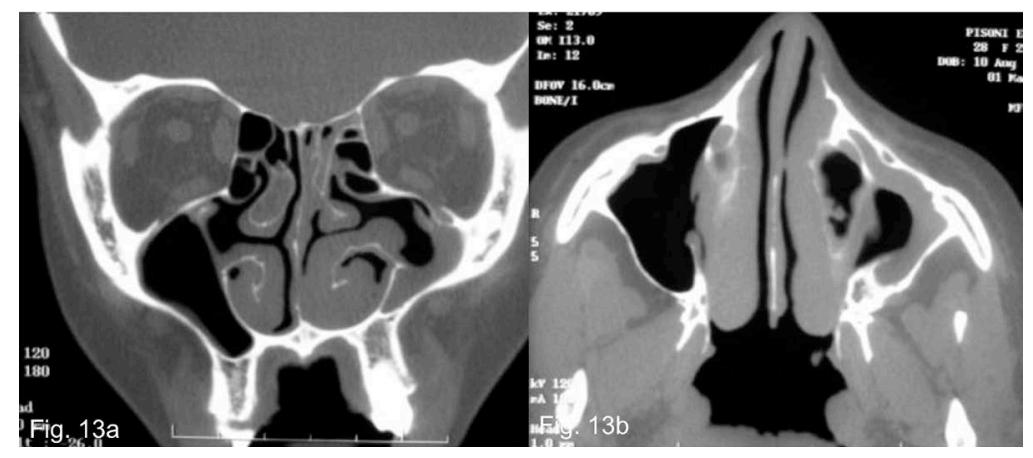

Figure 13. CT scan in coronal (a) and in axial (b) of a case of CMA with shrinkage of the left maxillary sinus due only to displacement of the medial antral wall.

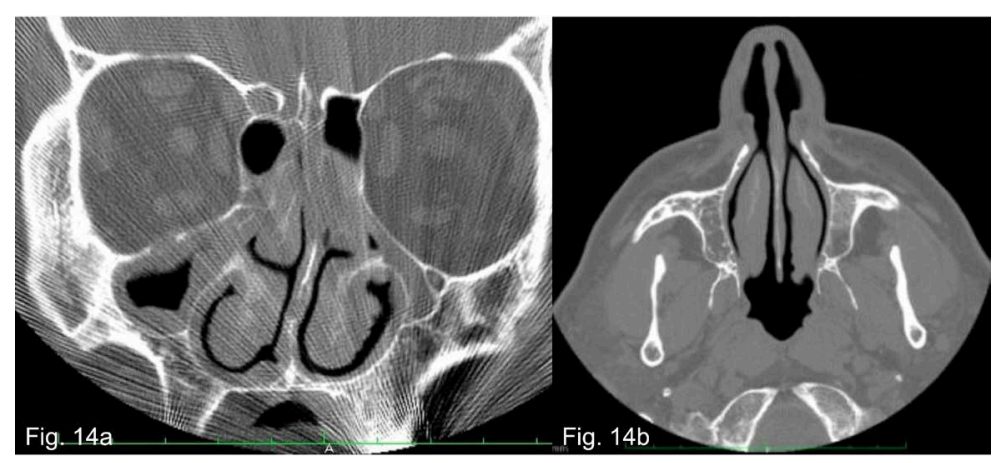

Figure 14. CT scan in coronal (a) and in axial (b) showing an atelectasic underdeveloped left maxillary sinus within an almost completely bone structured maxilla. 


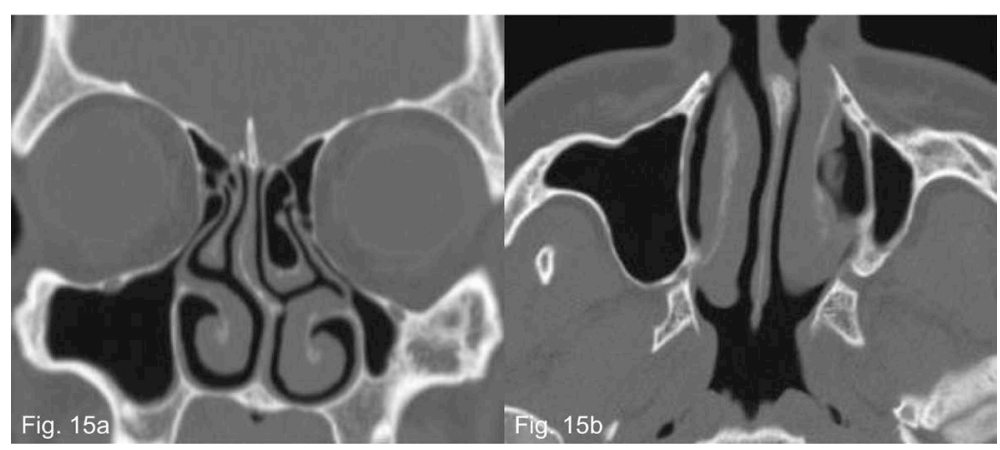

Figure 15. CT scan in coronal (a) and in axial (b) of a patient with aerated underdeveloped left maxillary sinus.

filling (Figure 15). CMA and SSS seem to represent different evolution stages of the same disease so that the absence of eye and face disorder in CMA doesn't ensure the patient from further bone remodelling. On the other hand appropriate surgical treatments have proven to provide recovery from sinus illness preventing any other evolution [3]. Ophthalmologist, ENT end OMFS when depicting CMA features should advice the patient that middle antrostomy is indicated [15].

\section{Conclusion}

We consider FESS the proper surgical treatment for SSS and CMA able to arrest the process of bone reabsorption by clearing the sinus mucous content and establishing physiological ventilation [15]-[17]. We recommend the functional endoscopic approach performing uncinectomy and calibration of the maxillary ostium. A skilled intervention includes trying not to let bare bone after mucosal stripping or cause injury to the very thin medial orbital wall. Carefully adoption of blunt hooks and micro-cutting instruments will allow this practice. We never observed re-expansion of maxillary sinus bony boundary after surgery.

\section{Consent}

Written informed consent was obtained from all the patients for publication of this article and accompanying images. All diagnostic and therapeutic procedures were performed with patients' consent and respecting ethical principles of our institutional and national committee.

\section{Competing Interests}

The authors declare that they have no competing interests regarding the publication of this article.

\section{Author's Contributions}

EE and PB were responsible for diagnosing and performing surgery on the patient. BP reviewed the patients' medical records in order to collect all the available information. PB and BP drafted and prepared the manuscript and were responsible in reviewing of the literature. EE and PB were involved in revising the article for intellectual details. All authors read and approved the final manuscript.

\section{References}

[1] Castelein, S., Cohen, M., Ayache, D. and Kalp, P. (2002) Atelectasis of the Maxillary Sinus: Report of a Case of Acute Onset. Revue de Laryngologie Otologie Rhinologie, 123, 99-102.

[2] Soparkar, C.N.S., Patrinely, J.R., Cuaycong, M.J., et al. (1994) The Silent Sinus Syndrome: A Rare Cause of Spontaneous Enophthalmos. Ophthalmology, 101, 772-778. http://dx.doi.org/10.1016/S0161-6420(94)31267-X

[3] Bossolesi, P., Autelitano, L., Brusati, R. and Castelnuovo, P. (2008) The Silent Sinus Syndrome: Diagnosis and Surgical Treatment. Rhinology, 46, 308-316.

[4] Monos, T., Levy, J., Lifshitz, T. and Puterman, M. (2005) The Silent Sinus Syndrome. (IMAJ) The Israel Medical Association Journal, 7, 333-335. 
[5] Vander Meer, J.B., Harris, G., Toohill, R.J. and Smith, T.L. (2001) The Silent Sinus Syndrome. A Case Series and Literature Review. Laryngoscope, 111, 975-978. http://dx.doi.org/10.1097/00005537-200106000-00008

[6] Hobbs, C.G.L., Saunders, W. and Potts, M.J. (2004) Spontaneous Enophthalmos: Silent Sinus Syndrome. The Journal of Laryngology \& Otology, 118, 310-312. http://dx.doi.org/10.1258/002221504323012102

[7] Buono, L.M. (2004) The Silent Sinus Syndrome: Maxillary Sinus Atelectasis with Enophthalmos and Hypoglobus. Current Opinion in Ophthalmology, 15, 486-489. http://dx.doi.org/10.1097/01.icu.0000142510.68451.32

[8] Illner, A., Davidson, H.C., Harnsberger, H.R. and Hoffman, J. (2002) The Silent Sinus Syndrome: Clinical and Radiographic Findings. American Journal of Roentgenology, 178, 503-506. http://dx.doi.org/10.2214/ajr.178.2.1780503

[9] Rose, G.E., Sandy, C., Halberg, L., et al. (2003) Clinical and Radiologic Characteristics of the Imploding Antrum, or “Silent Sinus" Syndrome. Ophthalmology, 110, 811-818. http://dx.doi.org/10.1016/S0161-6420(02)01993-0

[10] Boyd, J.H., Yaffee, K. and Holds, J. (1998) Maxillary Sinus Atelectasis with Enophthalmos. Annals of Otology, Rhinology, and Laryngology, 107, 34-39.

[11] Saetti, R., Emanuelli, E., Cutrone, C., Barion, U., Rimini, A., Giusti, F., Derosas, F., Narne, S. and Staffieri, A. (1998) The Treatment of Choanal Atresia. Acta Otorhinolaryngology of Italy, 18, 307-312.

[12] Bolger, W.E., Woodruff Jr., W.W., Moreshead, J. and Parsons, D. (1990) Maxillary Sinus Hypoplasia: Classification and Description of Associated Uncinate Process Hypoplasia. Otolaryngology_Head and Neck Surgery, 103, 759-765.

[13] Ong, L.Y. and Mc Nab, A.A. (2003) The Silent Sinus Syndrome: A Case with Normal Predisease Imaging. Orbit, 22, 161-164. http://dx.doi.org/10.1076/orbi.22.3.161.15623

[14] Hazan, A., LeRoy, A., Chevalier, E., Benzaken, J., Waisberg, A., Cymbalista, M., Adotti, E. and Peytral, C. (1998) Processus atélectasique du sinus maxillaire analyse des stades évolutifs à propos de 4 cas. Ann Otolaryngol Chir Cervicofac, 115, 367-372.

[15] Sciarretta, V., Pasquini, E., Tesei, F., Modugno, G.C. and Farneti, G. (2006) Endoscopic Sinus Surgery for the Treatment of Maxillary Sinus Atelectasis and Silent Sinus Syndrome. Journal of Otolaryngology, 35, 60-63. http://dx.doi.org/10.2310/7070.2005.4098

[16] Facon, F., Eloy, P., Brasseur, P. and Collet, S. (2006) The Silent Sinus Syndrome. European Archives of Oto-RhinoLaryngology, 263, 567-571. http://dx.doi.org/10.1007/s00405-006-0011-4

[17] Costa, F., Emanuelli, E., Robiony, M., Zerman, N., Polini, F. and Politi, M. (2007) Endoscopic Surgical Treatment of Chronic Maxillary Sinusitis of Dental Origin. Journal of Oral and Maxillofacial Surgery, 65, 223-228. http://dx.doi.org/10.1016/j.joms.2005.11.109 